\title{
Risultati della ricerca dei vuoti sotterranei con il metodo dei profili di resistivita in una zona delle fondazioni di un Viadotto
}

\author{
(Results in a research of burried cavilies by resistivity \\ profiles methods in the foundation's area of a Viaduct)
}

li. BRI\%\%OI.IRI (*)

Ricovuto il 15 (iennaio 197:)

\begin{abstract}
Rassusto. ... si riferise sui risultati ottenuti in una ricerea per ravita sotterranee. Fi stato impiegato un dispositivo a correnti foralizzate tipo (irandinetti. E stato effettuato un confronto pratico in campagna tra il dispositivo schlumberger a dispositivi tipo Grandinetti. I risultati della interpretazome effettuata confrontando lo enrve di campagna con gli andancenti teorice esistenti in biblegrafia e con quelli calcolati per l'oreasione, hanno permesso di individuare alcune eavita sotterrane dimostrando l'of. fieacia del dispositivo (iranelinetti.
\end{abstract}

SUMMARY. .. We refer on ohtained results in an investigation of burried cavities. We employed a "Grandinettis " clectrode array using focalised current. We have eompared directly on field sehlumberger array and Crandinettis clectrode array. The results of the interpretation, made with comparation method, have permited to find some buried cavities demonst rating the eflicacy of crandinetti"s electrode array.

\section{PREMGSNE}

Uno dei metodi geofisiei per la ricerea di vuoti soltermen a piecola profonditì $\dot{e}$, come noto, il metodo geoelettrico. In particolare

(*) Istituto di Geofisica Mineraria - Universiti degli Studi, Roma. 
rengono impiegati i metodi a resistivita e tra exsi, generalmente, quello dei profili di resistivita.

Fella presente nota vengono riferiti i risultati oftenuti, mediante lesernzione di profili di resistivita, nella licerea dei vuoti nella zona di fondazione del Viadot to "LXIntica " della Sora-Frosinone.

h'interesse dalla ricerca consiste nell'aver utilizato, nell'esecuzione dei prolili di resistività, un dispositivo a comenti focalizzate del tipo "Crandinetti " che per le sue caratteristiche geometriche e di campo permette di rilevare anomalie non facilmente indiviolubili con i dispositivi tradizionali.

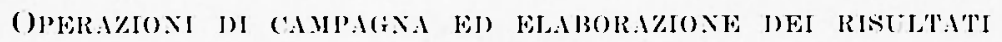

Nella zona oggetto dell'indagine esisteva il sospetto della flresenza di una coltivazione in sotterraneo di materiali vulcanici con moprieta pozzolaniche.

Si i pensato pertanto di aceertare loventuale presenza di vuoti, mediante l'esecuzione di profili di resistivita palralleli all'asse del viadotto e ad esso ortogonali. Trattandosi peraltro di una indagine preliminare, essa non i stata volta alla determinazione di tutti gli eventuali ruoti della zona ma piuttosto a stabilire la loro esistenza a al individuane be posizione nelle immediate vieinange del futuro asse stradale. Pertanto la zona investigata non risulta molto estesa, no i profili tropluo raveicinati. Nellindagine si sono utilizati il dispositivo Soldumberger e due dispositivi tipo Grandinetti (figura 1) allo scopo di ronfentarne i risultati.

\section{DISPOSITIVO SCHLUMBERGER}

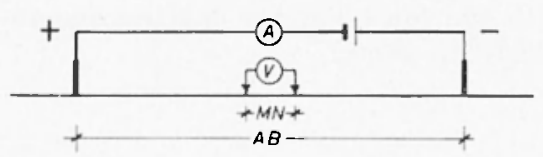

DISEOSITIVO GRAMOINETTI

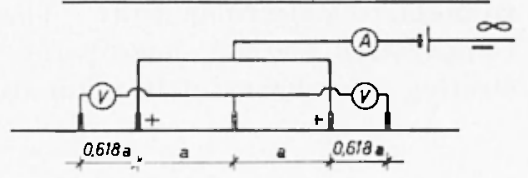

\section{DISPOSITIVO GRAMOIMETTI MODIFICATO}

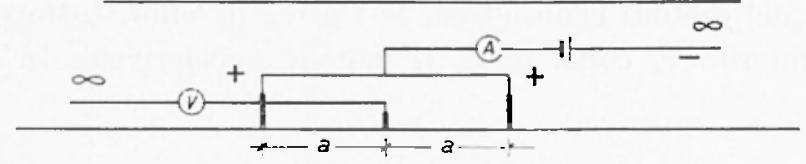

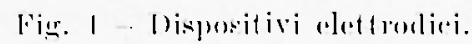


E stato utilizzato il dispositivo Glamblinetti tijo " $\mathrm{C}$ " (Grandinetti) (3) a nn dispositivo ali tipo trielettroslo con elettrodo di potenziale di riferimento all’infinito.

Lat seelta delle distanze elettrodiche i stata fatta sulla base della supposta profomblita degli eventuali vuoti e delle loro dimensioni probabili. Le notizie raceolte sul luogo da pessone ehe averano visto o avuto notizie indirette sui vuoti esistenti nella zona indicavano vuoti

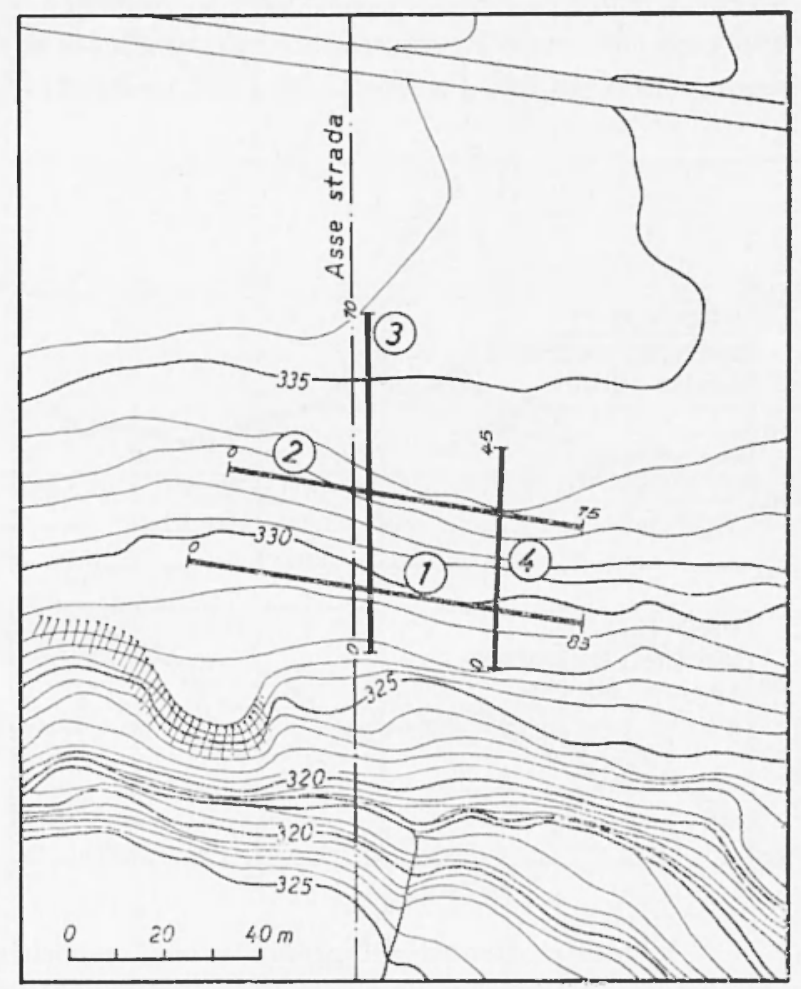

Fig. 2 - Ubicazione dei profili di resistivita.

con profondita del tetto variabili tra 1,5 e 5 m e dimensioni delle gallerie non superiori a $2-4 \mathrm{~m}$. In aceorslo con tali ordini di grandeze somo state fissate distanze elettroliche $a=3 \mathrm{ml}$ per i dispositivi Grandineti, mentre per quelli Sollumberger si sono adollate distanze $A B=6 \mathrm{~m}$ e $A B=10 \mathrm{~m}$. Isubicazione dei profili i indicala nella ligura 2. 
Il protilo 1 i stato ripetuto con diversi dispositivi e diverse distanze elettrodiche al fine di individuare la migliore distanza elettrodica e verificare la eftettiva utilità dei dispositivi Gandinetti $\left(^{3}\right)$.

I risultati ottenuti su tale profilo sono riportati nelle figure 3a a 3b per quanto riguarda i dispositivi Gmandinetti e sulla figura + per il clispositivo Schlumberger con distanze elettrodiche $A B=6 \mathrm{~m}$ e $A B=10 \mathrm{~m}$. Dei profili sono stati riportati solo i tratti più signifiativi.

Nella figura 4 sembra possibile accertare la presenza di anomalie collegabili con eventuali vuoti sotterranei. Per verificare d'altra parte che le variazioni siano comnesse a efletti anomali profondi e non at dis-

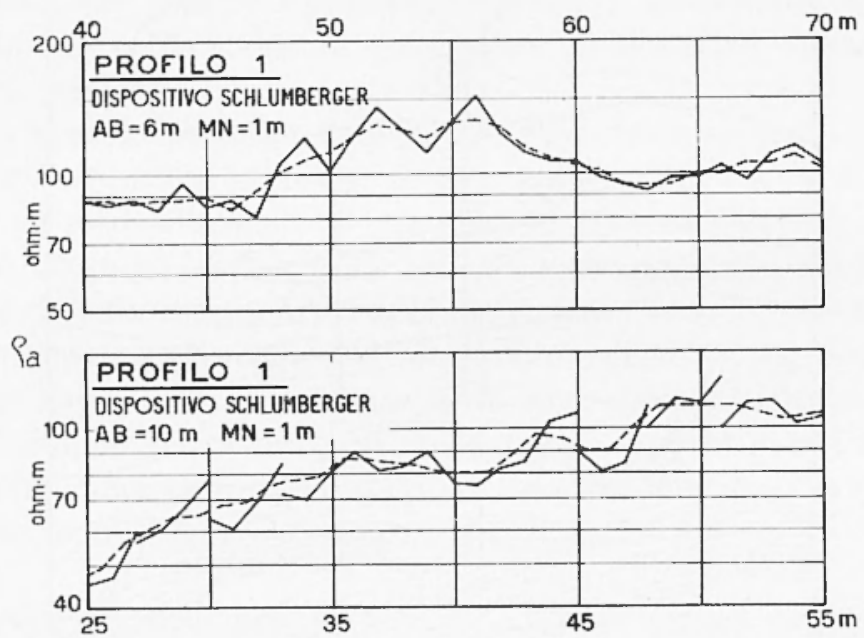

[Pir. + Risultati ottenuti sul pofolo l con rispositivo sehlumbererer. A tratteggio le medie di tre valori contigni, riferiti alla prostessiva centrale del smpupo.

omogeneità superficiali, è stata calcolata la media di 3 o più valori contigui attribuendone poi il valore alla progressiva centrale del grup)po. Cosi facenclo sembra pero accertato ohe la resistivita apparente varia con regolariti probabilmente solo a causa di variazionj laterali della resistivià del terreno. 


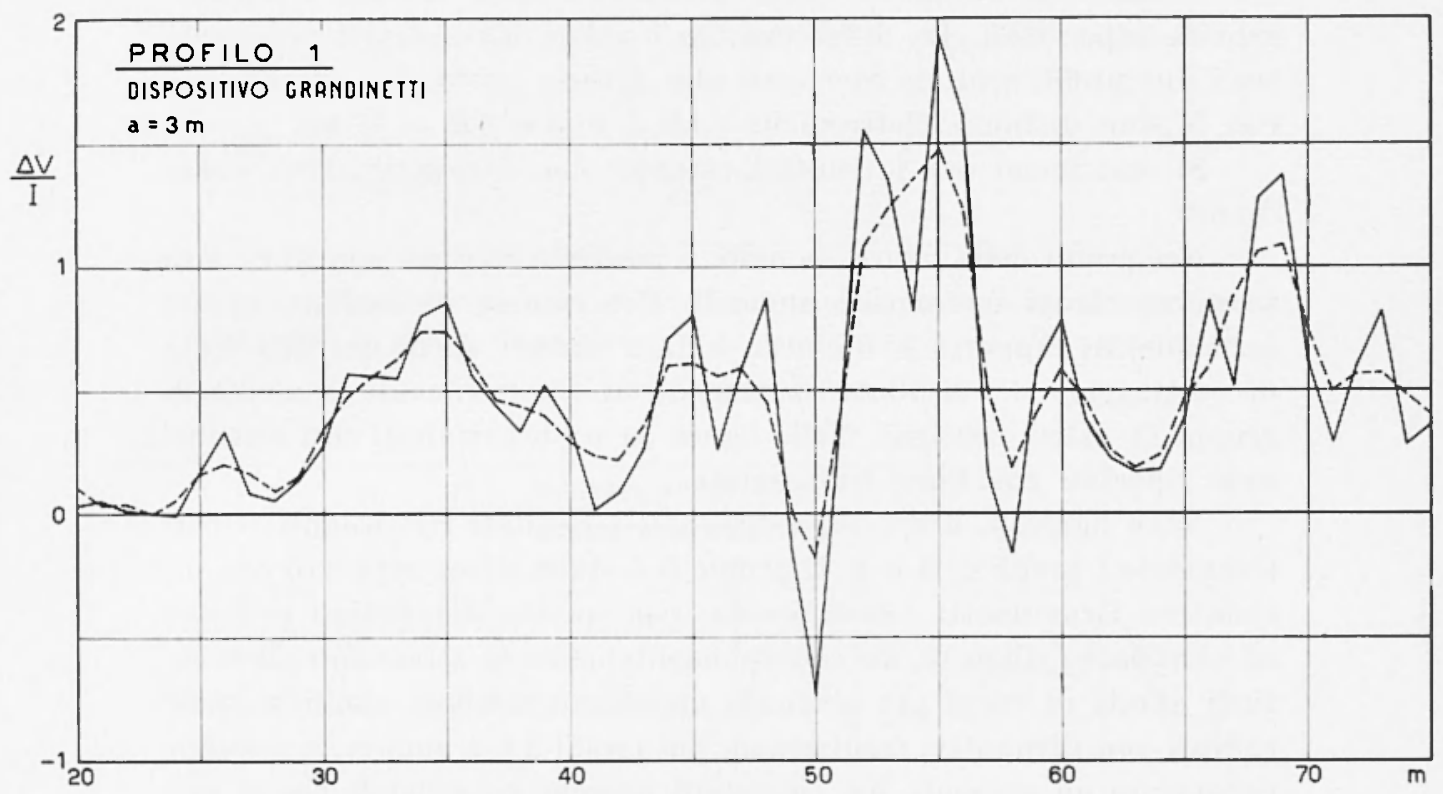

Fig. 3il - Risultati ottenuti sul profilo l con nn dispositivo Grandinetti. A trattegrgio le medie di tre valori eontigui, riferiti alla progressiva centrale del grmpluo.

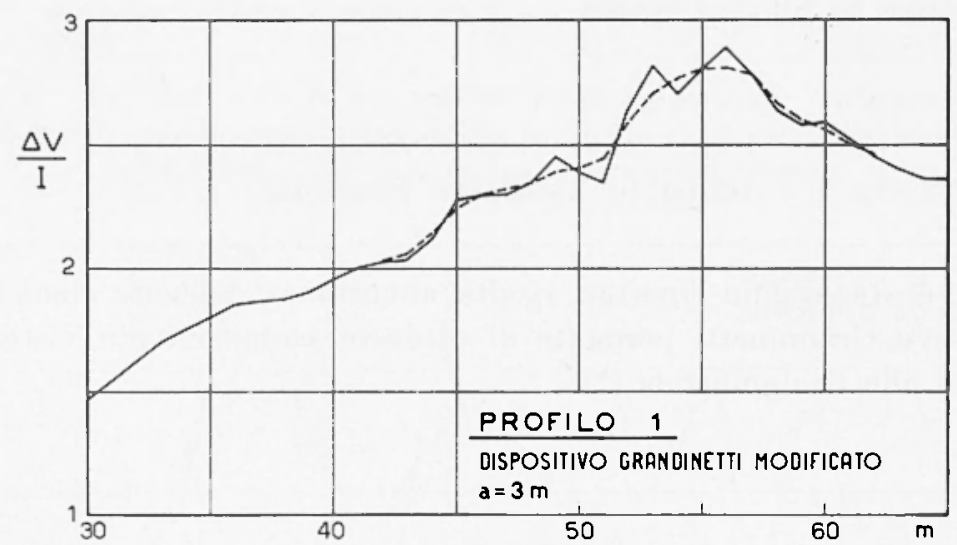

Fig. 31) - Risultati oftenuti sul profilo 1 con un dispositivo (irandinetti modificato. A tratteggio le medie di tre valori contigni, riferiti alla progressiva centrale del gruppo. 
Pertanto le variazioni sono presumibilmente dovute a disomogeneitis superficiali. La differenza tra i valori di resistivita apparente tra i dne profili sono da ascriversi alla diversa profondita investigata con le due distanze elettrodiche $(A B=6 \mathrm{~m}$ e $A B=-=10 \mathrm{~m})$.

Si esaminano ora i risultati ottenuti con dispositivi tipo Grandinetti.

Nei profili delle figure $3 a$ e $3 b$, è possibile rilevare con una rerta sicurezza alcuni andamenti anomali. Per cercare di esaltare queste anomalie, in rapporto ai rlisturbi, è stata tentata anche qui una sorta di "filtraggio " dei risultati sostituendo ai valori rilevati la merlia di gruppi di valori contigui. Nelle figure 3a e 3b i risultati cosi ottenuti sono riportati con linea tratteggiata.

Nelle figure 5,6 e 7 sono disegnati i risultati riguardanti rispettivamente i profili 2,3 e 4 . Il profilo 3 è stato altresi ripetuto con dispositivo Grandinetti "modificato "; con questo dispositivo si riesce ad eliminare i disturbi, ma corrispondentemente si attenuano gli eventrali efietti di corpi più profondi ottenendo risultati simili a quelli rilevati con dispositivi tradizionali. Sui profili 3 e 4 , inoltre, è possibile riscontrare un aumento dei contributi anomali superficiali, che si manifestano con variazioni notevoli delle difierenze di potenziale sn brevi spazi; si sono ottenute ciò variazioni con "frequenze " elevate indianti morleste dimensioni dei corpi ansa di tali anomalie, rispetto alle dimensioni del dispositivo. Sono stati anche qui calcolati i valori merli, come sopra indicato, che sembrano eliminare in gran parte le variazioni su breve distanza.

CONFRONTO F CALCOLO DI ANOMALIE TEORICIIF

Nei diagrammi riportati risulta abbastanza evidente come il dispositivo Grandinetti permette di ottenere variazioni più vistose rispetto allo schlumberger (*).

(*) Risultati opposti a quelli da noi rilevati in questo e in casi analoghli sono stati ottenuti da Armando (1). 


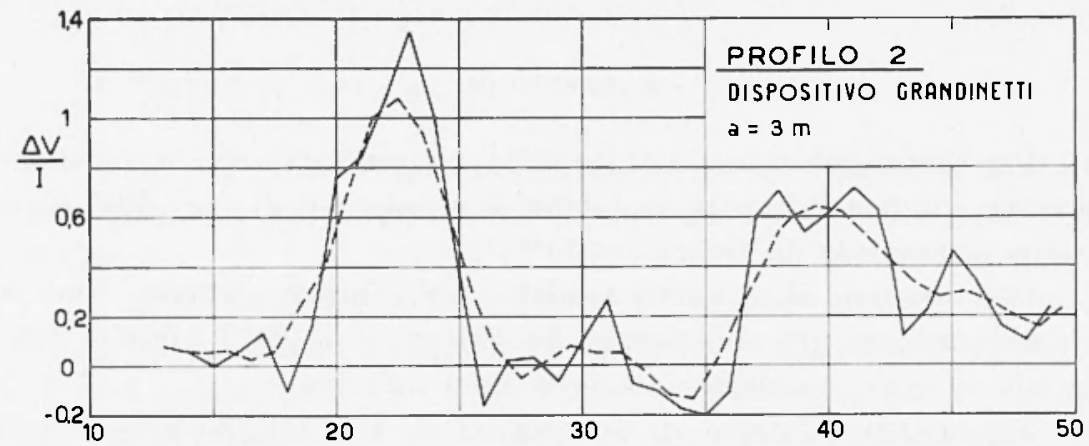

Fig. 5 - Risultati oftenuti sul profilo 2 con dispositivo (irandinetti. $A$ tratleggio le medie di quattro valori contigui, riferiti alla progressivat centrale del gruppo.
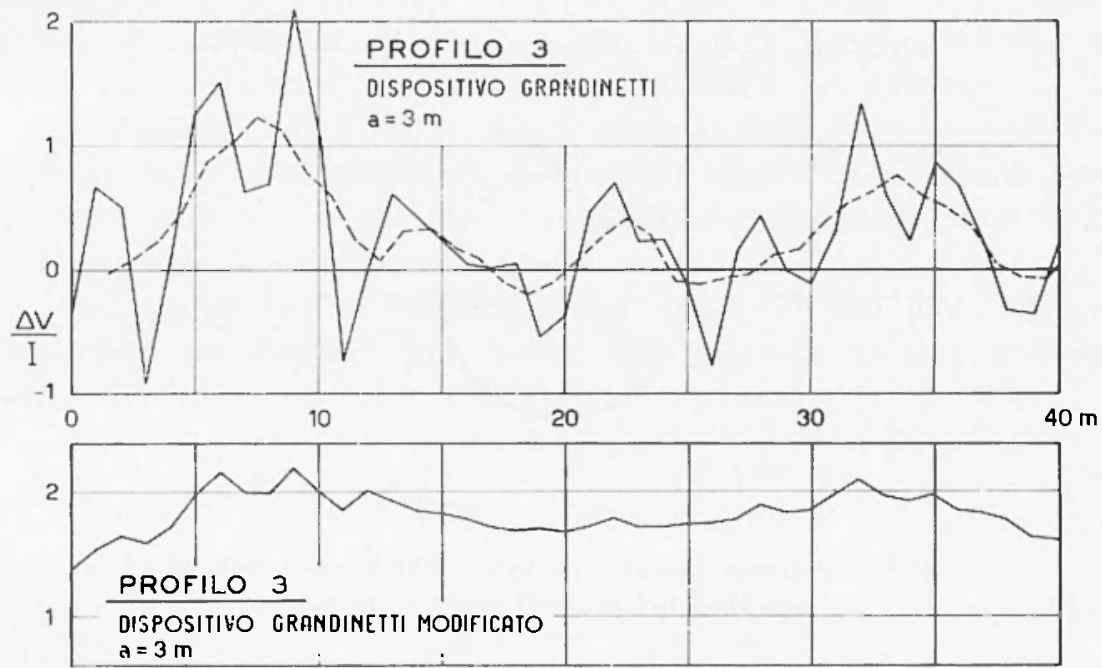

Fig. (; - Risultati ottenuti sul profilo 3 con dispositivo Cirandinetti e cirandinceti modificato. A tratteagrio le medie di quattro valori contigui. riferiti alla progressiva centrale del gruppou.

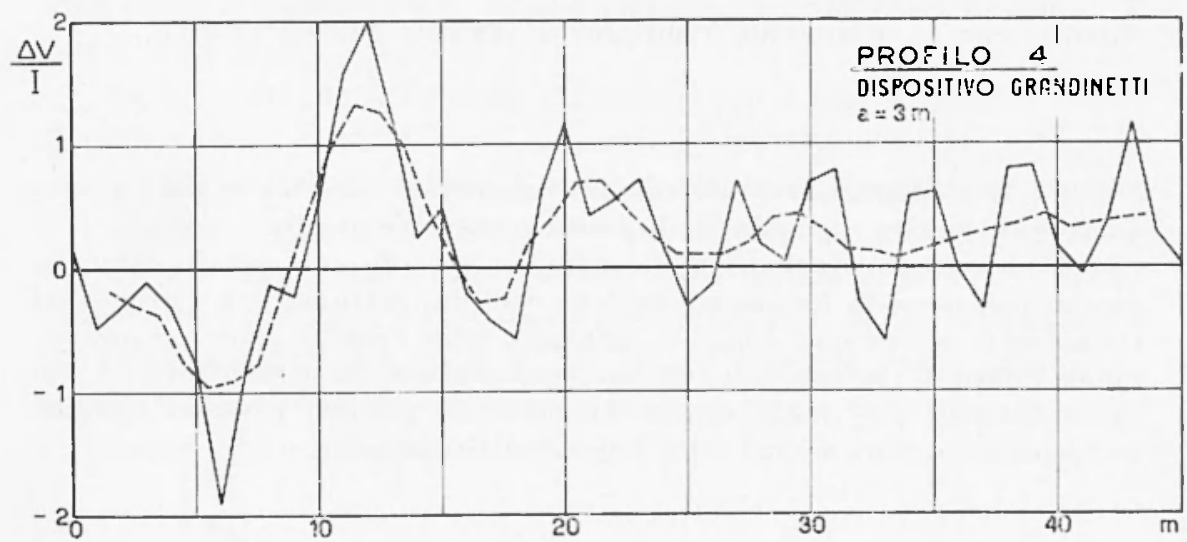

Jig. 7 . Risultati offenuti sul profilo 4 con dispositivo Grandinetti. A trat. fergio le medie di quattoo valori contigui, riferiti alla progressiva centrake del gruppo. 
Per l'interpretazione, i stato fatio un confronto ron le anomalie ricavate sperimentalmente in laboratorio nel aso di un cilindro resistivo orizzontale da Grandiretti(')(*).

Per ottenere altre curve teoriche per l'interputatione, oltre le schematizazioni gia considerate da Grandinetti per i cilindri orizzontali, si sono considerate anche le sfere resistive.

Supponiamo dunque di schematizaare i vuoti sottermanei con sfere poste anl man rerta profondita (lig. 8).

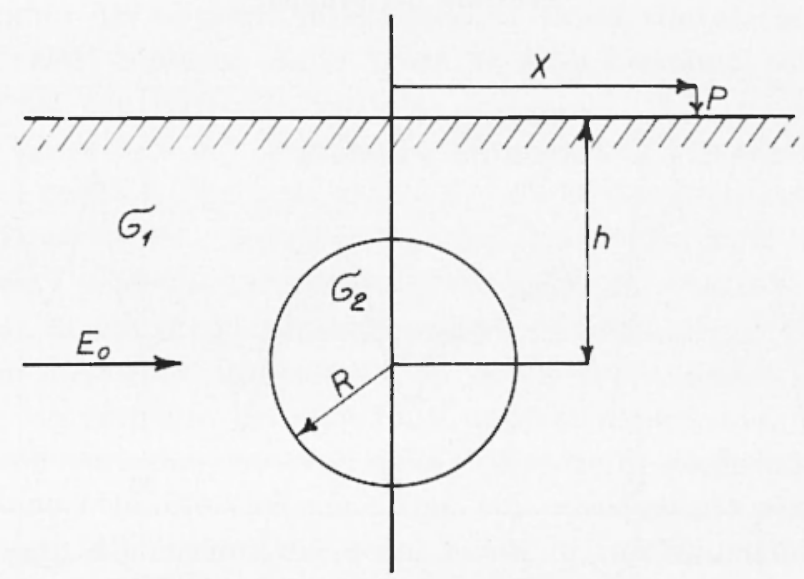

Fig. s - sehema geonetrien per il caleolo dei potenziali anomali nel aso di sfera resistiva.

In questo caso is possibile la soluzione matematica scegliendo un opportuno sistema di coobdinate (vedi Stucehi) $\left(^{6}\right)$ ovvero utilizzando la teoria delle immagini elettriche $\left({ }^{5}\right)$. Le soluzioni, ovviamente coinridenti, che si ottengono, risultano di difficile alcolo pratice.

(*) Il medesimo cano del cilindro orizzontale resistivo i stato risolto anche per quanto riguaded il disposition tipo Wonner $\left(^{7}\right)$.

In confronto qualitation dimostra, in accordo con quanto oftemuto

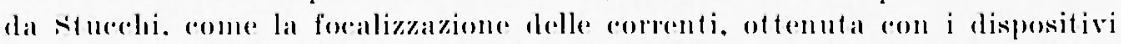
Grandinetti, abhia ma noterole influenza sulla "penetrazione". Confrontando infatti i risultat otfenuti sperinentalunente da (irandincelti (') con quelli calcolati per il dispositivo Wenuer (7) si possono notare i risultati neltanente migliori nel caso dei dispositivi Cimanlinetii. 
Sostanziali semplificazioni possono essere ottenute, con monleste approssimazioni nei risultati finali, introblucenclo alcune condizioni restrittive.

Ial sfera resistiva posta al di sotto del piano di campagna, genera rispetto ad esso, ma sua immagrine elettriea in posizione simmetrica. Nel caso semplificato che si considera, il potenziale elettrico is calcolato come somma dei due potenziali generati separatamente dalla sfera e dalla sua immagine. Si trascumono pereio gli effetti mutui tara le clue sfere; ciò non eomporta errori superiori al $10 \%$ sui valori caleolati finche risulta verificato che $h>1,3 R$, con $h$ profondita del centro della sfera medesima. Perciò si puo notare che non si hanno apprezabili errori fin quamo la parte superiore della ravita non si trova nelle immerliate vieinanze della superficie topografica.

Si suppone inoltre che il ampo elettrico generato possa ritenersi costante nella zona interessata dalla sfera; questa ipotesi risulta abbastanza rerificata se la sfera is suffecentemente clistante clai punti di immissione di corrente nel terreno.

Con queste ipotesi semplificative il potenziale sul piano $Z=0$ (superficie del terreno) puo essere cosi espresso (Grant e West par. $4 \cdot 5 \overline{5})(5)$ :

$$
\begin{aligned}
& V=-2\left(\frac{\sigma_{2}-\sigma_{1}}{\sigma_{2}+2 \sigma_{1}}\right) \frac{E_{o} R^{3} x}{\left(x^{2}+h^{2}\right)^{3 / 2}} \\
& V=-2\left(\frac{\sigma_{2}-\sigma_{1}}{\sigma_{2}+2 \sigma_{1}}\right) \frac{E_{v} R^{3} h}{\left(x^{2}+h^{2}\right)^{3 / 2}}
\end{aligned}
$$

dove $\sigma_{1}$ e $\sigma_{2}$ sono rispettivamente la conducibilita elettrica del tereno e della sfera di disturbo: $E_{0}$ e $E_{1}$ sono rispettivamente la componente orizzontale e verticale del ampo elettrico indisturbato calcolato al centro della sfera.

Sulla scorta clelle formule [1] e [\%], sono stati calcolati i profili anomali teorici oftenibili con rispositivi tipo Grandinetti.

Il alcolo rel potenziale i stato fatto per ma corrente erogata di 1 ampòre ponendo la resistivita del terreno pari a $0:=1$. I risultati di tali alcoli sono mppresentati nella figura 9 nel caso di una distanza elettrodica: $"=1$ e mggio della sfera $R=0$,is $a$, profomdita $h$ del corpo variabile tra $0,75 a$ e 2 . Fella figura 10 inoltre sono lappresentate altre due enre con profonclita e raggrio della sfera costanti a distanza olettroclica variabile. 
Coxchentoxi

Sulla scorla dei prolili teorici esistenti nella bibliografia crandinetti ('), e di quelli appositamente calcolati precendentemente con la schematizzazione della sfera eava (vedi ligure 9 e 10) is stata possibile una interpretazione per confronto con le anomalie rilevale nei profili di campagna. Tale confronto d risultato in alcuni casi alquanto diflicile in quanto nei profili di campagna mal certa importanza, come visto, assmmono le valiazioni su breve distanza imputabili certamente a disturbi superficiali e non già a disturbi più profondi.

Cercando pertanto di fare astrazione dagli andamenti locali e considerando le sole anomalie che investono un più ampio tratto del profilo sono state individuate sui profili stessi alcune zone per le quali si poteva presumere lexistenza di vuoti sotteranei. In conclusione sul profilo 1 sembrano esistere anomalie signiliative intorno alle progressive $26 ; 34,5 ; 50 ; 54 ;$ sul profilo 2 si sono individuate anomalie in eorrispondenza delle progressive 23 e 39,5 ; sul profilo 3 inline in corrispondenza della progressiva 32 sembra esistere un andamento anomalo.

Per accertare la presenza o meno di rnoti sotterranei in corrispondenza delle anomalie sopmandicate, sono stati eseguiti alcuni sondaggi meecanicei ad esse in corrispondenza. I risultati delle perforazioni posisono esisere sintetizzati nella seguente tabella.

'TABLILA

\begin{tabular}{|c|c|c|}
\hline P'rofilo & Progressivall & Vinoto \\
\hline $\begin{array}{l}1 \\
1 \\
1 \\
1 \\
2 \\
2 \\
3\end{array}$ & $\begin{array}{l}26 \\
34,5 \\
50 \\
54 \\
23 \\
39.5 \\
32\end{array}$ & 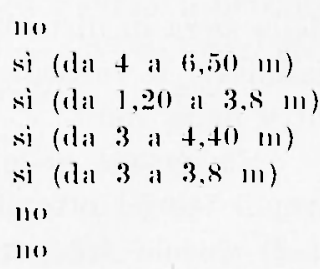 \\
\hline
\end{tabular}

is stata pertanto eonfermata lesistenza di rooti sotterranei in corrispondenza della zona investigata. Tali ruoti sembrano avere altezze medie di cira $2 \mathrm{~m}$ e profondita del letto variabili 1 ra $1,20 \mathrm{~m}$ e $1 \mathrm{~m}$ al di sotto del piano di ampagna. 


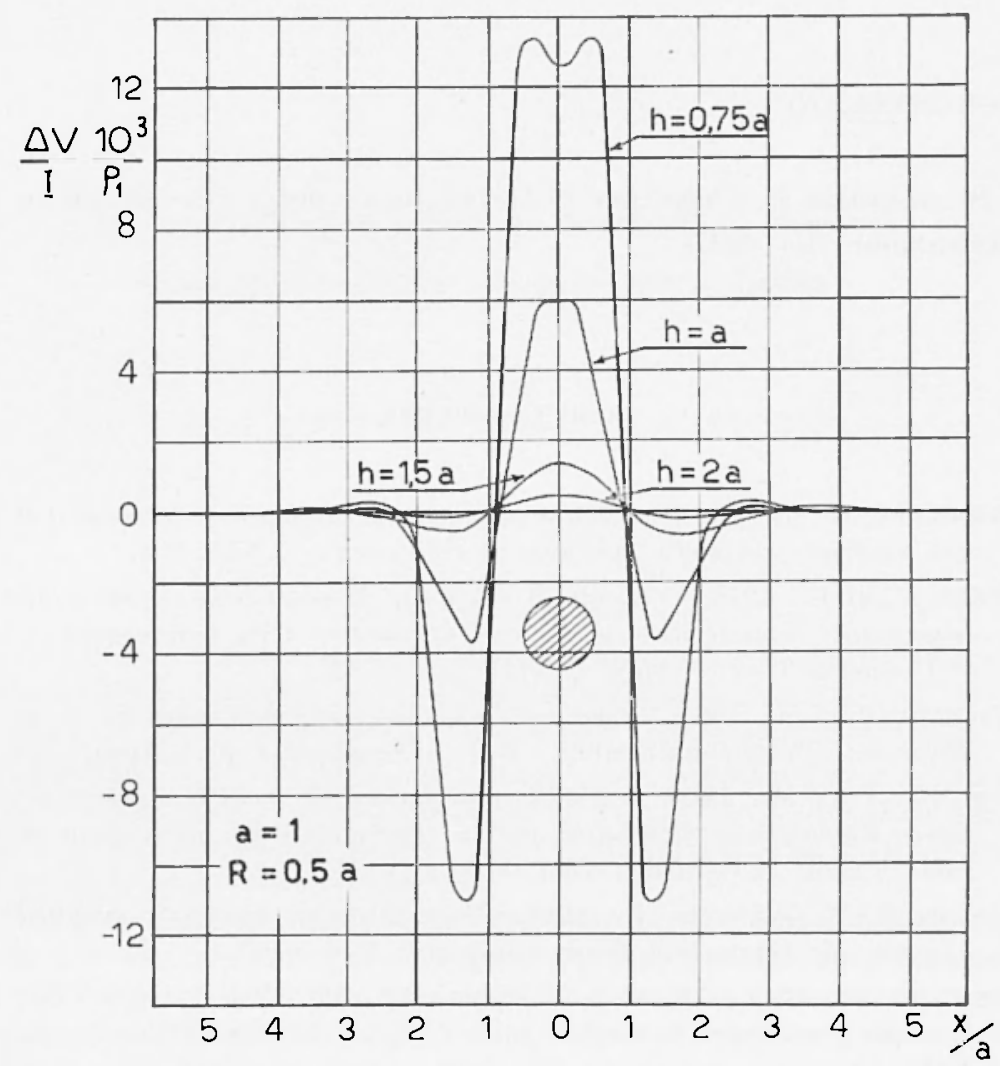

Pig. @ - Potenziali teorici caleolati nel aso eli sfera resistiva con dispositivo

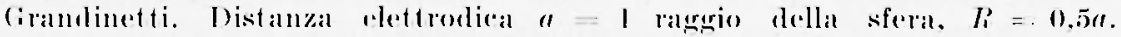
Profondita variabili tra $0,7 \overline{5}$ a e 2 a.

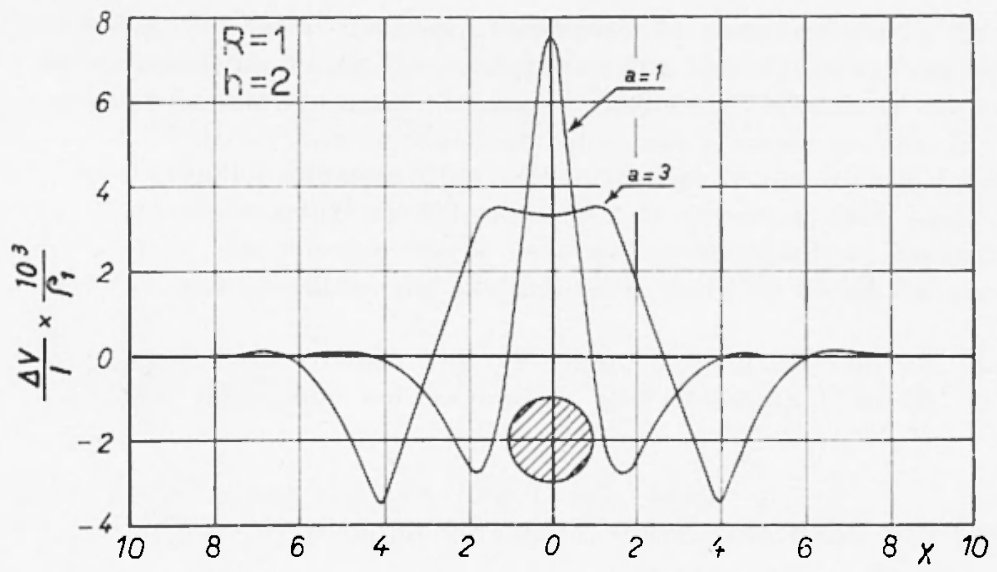

Yigr. 10 - Potenziali feorice calcolati nel raso di sfera resistiva con dispositivo Grandinetei nei duc rasi indirati in figura. 


\section{RIN(rRIZILMENTO}

Si ringrazia la Cassa per il Mezzogiorno per l'antorizzazione alla pubblicazione dei dati.

\section{BIBILIO(:RAFIA}

(1) Anuaxoo E., 1973 - Contribulo alla ricerea di cacilà sollerranee con melodi elellrici. "Rivista Italianla di Geofisica ", XXII, 3-4.

(2) Brizzodari F.. 1971 - Caleolo di anomalie di polenziale dorule a disturbi superficiali semisjerici rilecale con dispositivo hipo Grandinethi. "Boll. di Geofisica T'eor. C Appl. ", XIII, pp. 51-52.

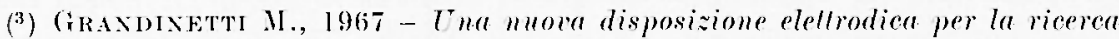
di corpi di limilale dimensioni. "Boll. di Geofisica T'eor. e Appl. ", IX, 35.

(1) GRAxDINETT II., 1968 - Risullati di prove su modelli relative ad $\mathrm{ma}$ muoce disposizione elellrodica per la ricerea di corpi di limilate dimensioni. "Boll. di Geofisical Teor. a Appl.", X. 39.

${ }^{(5)}$ Graxt F. S., WEsT (4. F., 1966 -Interpretation theory in applied geophysies. Me Graw-Ilill Book Company. New York.

(i) Strucen M., 1972-Ricerea del dispositieo eleltrodico ollimale nella prospesione geoelellrica di corpi sepolli. "Boll. di coofisiea Teor. "Appl.", XIV. 53.

(a) Vax Nostraxd R., Cook K., 1966 - Interpretation of resistivily dala. "Geological survey", Professional Paper 499. Vnited States Government Printing Office, Washington. 